\title{
Antimelanoma Differentiation-associated Gene 5 Dermatomyositis
}

DREW J.B. KURTZMAN, MD, Divisions of Dermatology and Rheumatology, The University of Arizona College of Medicine, Tucson, Arizona; MICHAEL WEINBLATT, MD, Division of Rheumatology, Immunology and Allergy, Brigham and Women's Hospital, Harvard Medical School, Boston, Massachusetts; RUTH ANN VLEUGELS, MD, MPH, Department of Dermatology, Brigham and Women's Hospital, Harvard Medical School, Boston, Massachusetts, USA. Address correspondence to Dr. D.J. Kurtzman, The University of Arizona, Dermatology, 1515 N. Campbell Ave., P.O. Box 245024 , Tucson, Arizona 85724, USA. E-mail: drewkurtzman@gmail.com. The Institutional Review Board of Brigham and Women's Hospital (IRB 2011P000047) approved this study. The patient whose images appear gave written informed consent. J Rheumatol 2017;44:850-1; doi:10.3899/jrheum.161427

Melanoma differentiation-associated gene 5 (MDA5) is an autoantigen target that has been described in individuals with dermatomyositis (DM). Anti-MDA5 autoantibodies can be detected in $10 \%-30 \%$ of cases of DM, and when present, confer a substantial risk of mortality, usually from interstitial lung disease (ILD). Serologic detection of anti-MDA5 antibodies correlates with a specific clinical phenotype ${ }^{1}$. In addition to the typical cutaneous signs of DM, patients with anti-MDA5 antibodies characteristically develop cutaneous

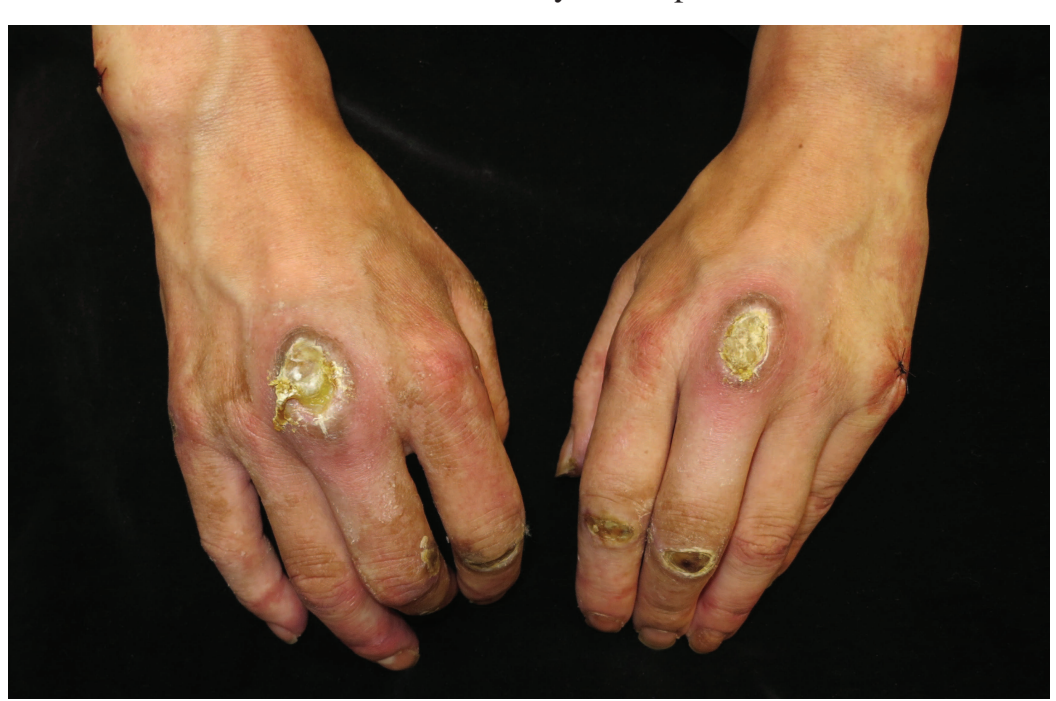

Figure 1. Cutaneous ulceration overlying Gottron papules in a patient with antimelanoma differentiation-associated gene 5 dermatomyositis. ulceration involving Gottron papules (Figure 1), the elbows, and digital pulp, as well as painful erythematous palmar papules (Figure 2), alopecia, panniculitis, and oral ulcers ${ }^{1}$. Anti-MDA5 DM is commonly clinically amyopathic, and arthritis frequently complicates the course of disease. Importantly, anti-MDA5 antibodies are strongly associated with ILD with a rapidly progressive course and poor overall survival $^{2,3}$. In a large case-control study examining the outcomes among individuals with DM with various myo-

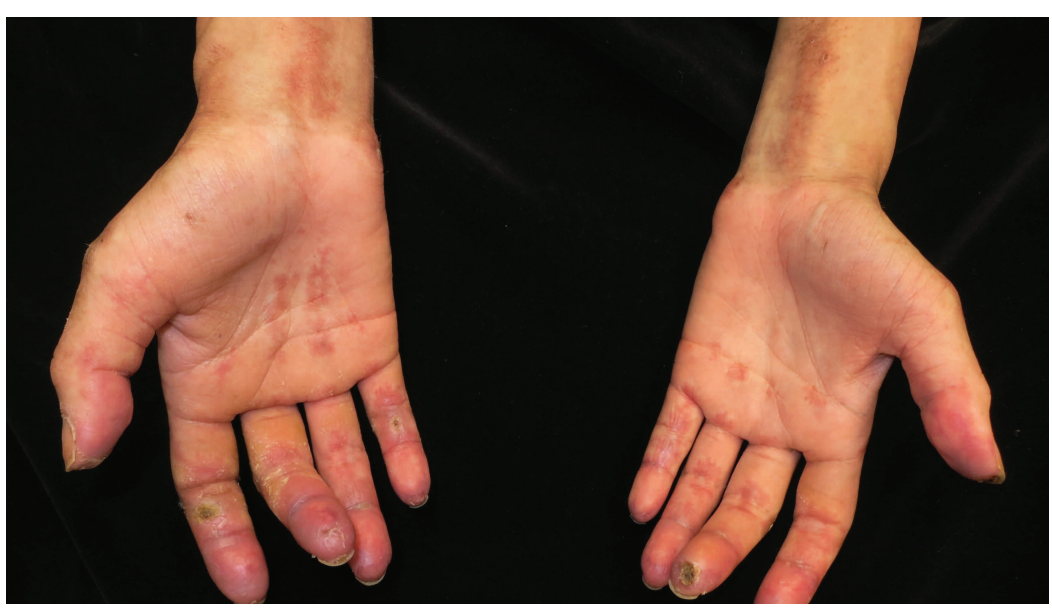

Personal non-commercial use only. The Journal of Rheumatology Copyright (c) 2017. All rights reserved. 
sitis-associated antibodies, those with anti-MDA5 antibodies displayed the lowest survival rates, even when compared with individuals with malignancy-associated DM, emphasizing the importance of identifying patients with MDA5 antibodies ${ }^{4}$. Anti-MDA5 DM is associated with a severe vasculopathy phenotype ${ }^{1}$, which may explain the tissue ischemia and infarction characteristically observed in the skin. Recognition of the clinical features of anti-MDA5 DM is imperative to facilitate an early diagnosis and allow for appropriate pulmonary screening and the institution of therapies that may reduce the morbidity and substantial risk of mortality associated with the disorder.

\section{REFERENCES}

1. Fiorentino D, Chung L, Zwerner J, Rosen A, Casciola-Rosen L. The mucocutaneous and systemic phenotype of dermatomyositis patients with antibodies to MDA5 (CADM-140): a retrospective study. J Am Acad Dermatol 2011;65:25-34.
2. Moghadam-Kia S, Oddis CV, Sato S, Kuwana M, Aggarwal R. Anti-melanoma differentiation-associated gene 5 is associated with rapidly progressive lung disease and poor survival in US patients with amyopathic and myopathic dermatomyositis. Arthritis Care Res 2016;68:689-94.

3. Chen Z, Cao M, Plana MN, Liang J, Cai H, Kuwana M, et al. Utility of anti-melanoma differentiation-associated gene 5 antibody measurement in identifying patients with dermatomyositis and a high risk for developing rapidly progressive interstitial lung disease: a review of the literature and a meta-analysis. Arthritis Care Res 2013;65:1316-24.

4. Hamaguchi Y, Kuwana M, Hoshino K, Hasegawa M, Kaji K, Matsushita T, et al. Clinical correlations with dermatomyositis-specific autoantibodies in adult Japanese patients with dermatomyositis: a multicenter cross-sectional study. Arch Dermatol 2011;147:391-8. 